there is a much shorter solution of the problem by partial derivatives than the one which I gave as Solution 2. The solution is as follows:If $y$ and $z$ are the independent variables we have, since

$$
\begin{aligned}
\frac{\partial x}{\partial z} & =\frac{1}{p}, \quad \frac{\partial x}{\partial y}=-\frac{q}{p}, \\
\frac{\partial^{2} x}{\partial y \partial z}=\frac{\partial}{\partial y}\left(\frac{1}{p}\right) & =-\frac{1}{p^{2}}\left\{\frac{\partial p}{\partial y}+\frac{\partial p}{\partial x} \frac{\partial x}{\partial y}\right\} \\
& =-\frac{1}{p^{2}}\left\{s+r\left(-\frac{q}{p}\right)\right\} \\
& =\frac{1}{p^{3}}(r q-s p),
\end{aligned}
$$

which gives the result required.

Since my Solution 2 was, quite unintentionally, rather unfair to the method of partial derivatives, I feel that I ought to draw attention to this shorter solution.

The fact that the above solution is merely shorter than the one which I gave does not however detract from the practical advantages of the differential method. Any experienced teacher knows that the step which presents real difficulty to the beginner is the obtaining of equation (1) above. Although in the case of the example which I happened to choose for illustration (and it may not have been the best for the purpose) the above solution by partial derivatives happens to be quite as short as the solution by differentials, the fact remains that, while the technique of differentiation, when once understood, is almost "fool-proof," the pitfalls for the beginner in the solution given above are well known to every teacher of the subject. While the solution of a problem by partial derivatives may be quite a difficult piece of manipulation, exactly the same technique is required for the solution of a problem by differentials, however simple or complicated the problem in question may happen to be.

\title{
On pedal tetrahedra
}

By R. T. Robinson.

1. In a tetrahedron $A B C D$ with its opposite edges perpendicular there are two tetrahedra which can be described as pedal tetrahedra.

(1) the tetrahedron $A_{0} B_{0} C_{0} D_{0}$ where these points are the feet of the perpendiculars from $A B C D$ on to the faces $B C D$. $A C D$.... called here the face-pedal tetrahedron. 
(2) the tetrahedron $A_{1} B_{1} C_{1} D_{1}$ formed in this way:-if $O$ is the ortho-centre of the tetrahedron $A B C D$, three straight lines $P P_{1}, Q Q_{1} . R R_{1}$ can be drawn through $O$ to meet the edges $A D . B C$ in $P P_{1}$, the edges $B D . C A$ in $Q Q_{1}$ and the edges $C D . A B$ in $R R_{1}$.

The planes $P Q R, P Q_{1} R_{1}, Q R_{1} P_{1}, R P_{1} Q_{1}$ are the faces $A_{1} B_{1} C_{1}$. $B_{1} C_{1} D_{1} \cdot A_{1} C_{1} D_{1} \cdot A_{1} B_{1} D_{1}$ of another tetrahedron $A_{1} B_{1} C_{1} D_{1}$ called here the edge-pedal tetrahedron of the tetrahedron $A B C D$. In this tetrahedron $A_{1} B_{1} C_{1} D_{1} O$ is the centre of its inscribed sphere and $A B C D$ are the centres of the e-scribed spheres opposite to $A_{1} B_{1} C_{1} D_{1}$ respectively: this can be proved as follows:-

2. $Q R \cdot Q_{1} R_{1}$ intersect at $S$ on $B C$ where $\left(B P_{1} C S\right)=-1$.

The planes $P Q R . P Q_{1} R_{1}$, i.e. the planes $A_{1} B_{1} C_{1} . B_{1} C_{1} D_{1}$, intersect in the straight line $B_{1} C_{1}$, therefore $B_{1} C_{1}$ passes through $S$, i.e. $B C$ and $B_{1} C_{1}$ are in the same plane, $A D$ is perpendicular to $P P_{1}$ and $B C$, therefore any plane through $A D$ is perpendicular to the plane $P B C$, therefore the plane $B_{1} C_{1} D$ is perpendicular to the plane $P B C$. Taking the four planes passing through $B_{1} C_{1}$, namely the planes $B_{1} C_{1} D$, $A_{1} B_{1} C_{1}$ (the plane $P Q R$ ). $B_{1} C_{1} O$ (the plane $B C B_{1} C_{1}$ ). $B_{1} C_{1} D_{1}$ (the plane $P Q_{1} R_{1}$ ), the straight line $A B$ cuts these four planes at $A X B R_{1}$ respectively, but $\left(A R_{1} B X\right)=-1$, and the planes $B_{1} C_{1} D . B_{1} C_{1} O$ are perpendicular, therefore the planes $B_{1} C_{1} D . B_{1} C_{1} O$ are the internal and external bisectors of the angle between the planes $A_{1} B_{1} C_{1}, B_{1} C_{1} D_{1}$, i.e. the planes bisecting the angles between the planes $A_{1} B_{1} C_{1} . B_{1} C_{1} D_{1}$ pass through $O$ and $D$.

Similarly the planes bisecting the angles between the planes $A_{1} B_{1} C_{1} \cdot A_{1} C_{1} D_{1}$ and the planes bisecting the angles between the planes $A_{1} B_{1} C_{1}, A_{1} B_{1} D_{1}$ pass through $O$ and $D$. Therefore $O$ and $D$ are the centres of the spheres inscribed in the tetrahedron $A_{1} B_{1} C_{1} D_{1}$. Similarly $A B C$ are the centres of spheres inscribed in the tetrahedron $A_{1} B_{1} C_{1} D_{1}$.

3. The coordinates of $O$ are

$$
-\frac{6 V \rho^{2}}{A\left(b^{2}+c^{2}-a^{2}\right)} \cdot-\frac{6 V \rho^{2}}{B\left(a^{2}+c^{2}-b^{2}\right)} \cdots-\frac{6 V \rho^{2}}{D\left(e^{2}+f^{2}-a^{2}\right)}
$$

where $\rho$ is the radius of the self-polar sphere of the tetrahedron $A B C D$ and $-576 V^{2} \rho^{2}=\left(b^{2}+c^{2}-a^{2}\right)\left(a^{2}+c^{2}-b^{2}\right)\left(a^{2}+b^{2}-c^{2}\right)\left(e^{2}+f^{2}-a^{2}\right)$ and the equation of $B_{1} C_{1} D_{1}$ is

$-A a\left(b^{2}+c^{2}-a^{2}\right)+B \beta\left(a^{2}+c^{2}-b^{2}\right)+C \gamma\left(a^{2}+b^{2}-c^{2}\right)+D \delta\left(e^{2}+f^{2}-a^{2}\right)=0$. 
This equation can be put in the form

$$
2\left(c^{2} B \beta+b^{2} C \gamma+d^{2} D \delta\right)-\left(b^{2}+c^{2}-a^{2}\right) \Sigma A \alpha=0,
$$

but the equation of the tangent plane at $A$ to the sphere $A B C D$ is

$$
c^{2} B \beta+b^{2} C \gamma+d^{2} D \delta=0,
$$

therefore $B_{1} C_{1} D_{1}$ is parallel to the tangent plane at $A$ to the sphere $A B C D$.

Similarly $A_{1} C_{1} D_{1}$ is parallel to the tangent plane at $B$, and so on.

i.e. the edge-pedal tetrahedron $A_{1} B_{1} C_{1} D_{1}$ is similar and similarly situated to the tetrahedron $A_{3} B_{3} C_{3} D_{3}$ formed by the tangent planes to the sphere $A B C D$ at the points $A B C D$.

4. To find the radii of these inscribed spheres.

It can be proved that the volume of $O P Q R=-\frac{\rho^{2}\left(e^{2}+f^{2}-a^{2}\right)^{2}}{2 d^{2} e^{2} f^{2}}$ and that the area of the $\triangle P Q R=\frac{3 V \rho_{1}\left(e^{2}+f^{2}-a^{2}\right)^{2}}{2 d^{2} e^{2} f^{2}}$ where $\rho_{1}$ is the radius of the sphere $A B C D$, therefore the perpendicular from $O$ on to the plane $P Q R$, i.e. the plane $A_{1} B_{1} C_{1}=-\frac{\rho^{2}}{\rho_{1}}$. Similarly it can be shewn that the lengths of the perpendiculars from $O$ on to the planes $B_{1} C_{1} D_{1} \cdot A_{1} C_{1} D_{1} \cdot A_{1} B_{1} D_{1}$ are each equal to $-\frac{\rho^{2}}{\rho_{1}}$, this is therefore the radius of the inscribed sphere.

5. It can be shewn that the volume of $D P Q R=\frac{V\left(e^{2}+f^{2}-a^{2}\right)^{3}}{\delta d^{2} e^{2} f^{2}}$ and that the perpendicular from $D$ on to the plane

$$
P Q R \text { or } A_{1} B_{1} C_{1}=\frac{e^{2}+f^{2}-a^{2}}{4 \rho_{1}}
$$

$=$ the length of the perpendiculars on to $B_{1} C_{1} D_{1} \cdot A_{1} C_{1} D_{1} \cdot A_{1} B_{1} D_{1}$

$=$ the radius of the $e$-scribed sphere opposite $D$.

The radii of the $e$-scribed spheres opposite $A_{1} B_{1} C_{1}$ can be proved in the same way to be equal to $\frac{b^{2}+c^{2}-a^{2}}{4 \rho_{1}}, \frac{a^{2}+c^{2}-b^{2}}{4 \rho_{1}}, \frac{a^{2}+b^{2}-c^{2}}{4 \rho_{1}}$.

6. The coordinates of $O$ (see section 3) can also be put in the form

$$
\frac{3\left(V-2 V_{1}\right)}{2 A} \cdots \frac{3\left(V-2 V_{4}\right)}{2 D}
$$

where $V_{1} V_{2} V_{3} V_{4}$ are the volumes of the tetrahedra $O_{1} B C D, O_{1} A C D$, $O_{1} A B D, O_{1} A B C, O_{1}$ being the centre of the sphere $A B C D$. 
Hence

$\left(b^{2}+c^{2}-a^{2}\right)\left(V-2 V_{1}\right)=\ldots .=\ldots=\left(e^{2}+f^{2}-a^{2}\right)\left(V-2 V_{4}\right)=-4 V \rho^{2}$, therefore the radii of the inscribed spheres centres $A B C D$ can be put in the form $\frac{V}{V-2 V_{1}}\left(-\frac{\rho^{2}}{\rho_{1}}\right) \cdots \frac{V}{V-2 V_{4}} \cdot\left(-\frac{\rho^{2}}{\rho_{1}}\right)$ from which we see that the sum of the reciprocals of the radii of the inscribed spheres opposite $A B C D=-\frac{2 \rho_{1}}{\rho^{2}}=$ twice the reciprocal of the radius of the inscribed sphere centre $O$. The three remaining inscribed spheres have radii equal to $\pm \frac{V}{V_{1}+V_{4}-V_{2}-V_{3}} \cdot\left(-\frac{\rho^{2}}{\rho_{1}}\right)$ etc.

7. If in the tetrahedron $A_{1} B_{1} C_{1} D_{1}$ the areas of the faces opposite $A_{1} B_{1} C_{1} D_{1}$ are represented by $A_{1} B_{1} C_{1} D_{1}$ we get from the preceding $\frac{-A_{1}+B_{1}+C_{1}+D_{1}}{A_{1}+B_{1}+\overline{C_{1}+D_{1}}}=\frac{V-2 V_{1}}{V}$ or $A_{1}=\frac{V_{1}}{V}\left(A_{1}+B_{1}+C_{1}+D_{1}\right)$ etc. i.e. $A_{1}: B_{1}: C_{1}: D_{1}=V_{1}: V_{2}: V_{3}: V_{4}$.

8. Since the edge-pedal tetrahedron $A_{1} B_{1} C_{1} D_{1}$ is similar and similarly situated to the tetrahedron $A_{3} B_{3} C_{3} D_{3}$ formed by the tangent planes at $A B C D$ to the sphere $A B C D$ the areas of the faces of $A_{3} B_{3} C_{3} D_{3}$ are proportional to $V_{1} V_{2} V_{3} V_{4}$ and the perpendiculars from $A B C D$ respectively on to the faces $B_{1} C_{1} D_{1} \ldots A_{1} B_{1} C_{1}$ of the edge-pedal tetrahedron pass through $O_{1}$ the centre of the sphere $A B C D$, hence $\cos A_{1} B_{1}=\frac{c^{2}}{2 \rho_{1}^{2}}-1$ etc. and $\cos A_{1} B_{1}+\cos C_{1} D_{1}=\cos A_{1} C_{1}+\cos B_{1} D_{1}=\cos B_{1} C_{1}+\cos A_{1} D_{1}$ $=\frac{a^{2}+d^{2}}{2 \rho_{1}^{2}}-2=-\frac{2 \rho^{2}}{\rho_{1}^{2}}$, and since $A_{1} B_{1} C_{1} D_{1}$ are proportional to $V_{1} V_{2} V_{3} V_{4}$ the relation $A_{1}=B_{1} \cos A_{1} B_{1}+C_{1} \cos A_{1} C_{1}+D_{1} \cos A_{1} D_{1}$ becomes

$$
\begin{aligned}
& V_{1}=V_{2}\left(\frac{c^{2}}{2 \rho_{1}^{2}}-1\right)+V_{3}\left(\frac{b^{2}}{2 \rho_{1}^{2}}-1\right)+V_{4}\left(\frac{d^{2}}{2 \rho_{1}^{2}}-1\right) \\
& \text { or } c^{2} V_{2}+b^{2} V_{3}+d^{2} V_{4}=2 V \rho_{1}^{2} .
\end{aligned}
$$

9. Since the radius of the inscribed sphere centre $O$ of the edgepedal tetrahedron $A_{1} B_{1} C_{1} D_{1}$ equals $-\frac{\rho^{2}}{\rho_{1}}$ and the radius of the inscribed sphere centre $O_{1}$ of the tetrahedron $A_{3} B_{3} C_{3} D_{3}$ equals $\rho_{1}$ the length of any edge of the tetrahedron $A_{1} B_{1} C_{1} D_{1}$ is to the length of the corresponding edge of the tetrahedron $A_{3} B_{3} C_{3} D_{3}$ as $-\rho^{2}$ is to $\rho_{1}^{2}$, or $A_{1} B_{1}: A_{3} B_{3}=-\rho^{2}: \rho_{1}^{2}$, and so on. 
viii

The centre of perspective for the two tetrahedra $A_{1} B_{1} C_{1} D_{1}: A_{3} B C_{3} D_{3}$ is the point where coordinates are $\alpha=\frac{1}{\rho_{1}^{2}+\rho^{2}} \cdot\left[\rho^{2} \cdot \frac{3 V_{1}}{A}+\rho_{1}^{2} \cdot \frac{3\left(V-2 V_{1}\right)}{2 A}\right]$ etc.

This point is the pole of the plane whose equation is $\Sigma A a\left(b^{2}+c^{2}-a^{2}\right)=O$ with respect to the sphere circumscribing the tetrahedron $A B C D$.

\title{
115 Shobnall Road, Burton-on-Trent.
}

\section{Some properties of the paraboloid $z=x^{2}+y^{2}$}

\author{
By D. Pedoe, Ph.D.
}

In a recent paper ${ }^{1}$, I showed how the properties of algebraic systems of circles in the $(x, y)$ plane could be investigated by means of a representation in which to the circle $x^{2}+y^{2}-2 p x-2 q y+r=0$ there corresponds the point $(p, q, r)$ in space of three dimensions. The plane of $(x, y)$ may be considered to lie in the space $(x, y, z)$, so that the centre of the mapped circle is the orthogonal projection of the representative point.

Point-circles, or circles of zero radius, are mapped by points on the paraboloid of revolution $z=x^{2}+y^{2}$, and this quadric, which we call $\Omega$, plays a fundamental part in the representation. An algebraic curve $C$ in the three-dimensional space $S_{3}$ represents an algebraio system of circles in the $(x, y)$ plane, and it was shown that the envelope of this system of circles is found by projecting orthogonally on to the $(x, y)$ plane the curve in which the polar lines with regard to $\Omega$ of the tangents to $C$ meet $\Omega$.

This representation offers a convenient method for obtaining what has recently been called the "circle-tangential equation" of a given plane curve ${ }^{2}$. Suppose we are given a plane algebraic curve, of equation $f(x, y)=0$. This curve is touched by an infinity of circles through the point $(0,0)$. If such a circle is $x^{2}+y^{2}-2 p x-2 q y=0$, a relation $g(p, q)=0$ holds; i.e. the centres of these contact circles lie on an algebraic curve of equation $g(x, y)=0$. This is called the circle-tangential equation of the given curve.

As a trivial example, the circle-tangential equation of a point $(a, b)$ is that of the perpendicular bisector of the join of this point to 